\title{
Patterning of thin film strain gauges on 3D-surfaces
}

\author{
O. Suttmann, J.F. Duesing, J. Koch, U. Stute, L. Overmeyer \\ Laser Zentrum Hannover e.V., Hollerithallee 8, 30419 Hannover, Germany, \\ o.suttmann@Izh.de
}

\begin{abstract}
:
Metal thin film strain gauges applied directly on the surface of components offer good long term stability even in harsh environments and at elevated temperatures. They consist of vacuum deposited isolation and sensing films. A direct-write laser patterning process based on picosecond laser pulses has been developed to overcome the restriction to 2D-surfaces caused by photolithographic patterning. We present results of tensile test of strain sensors patterned in v-notches milled in aluminum samples. The thermal coefficient of resistance (TCR) and the gauge factor are not affected by Laser patterning of conductor widths of $13 \mu \mathrm{m}$ and larger. Therefore, the developed process is suitable to pattern miniaturized thin film strain gauges on 3D-surfaces.
\end{abstract}

Due to the exploration of 3D-surfaces, the prerequisite for new sensing methods has been created to develop new applications which require the patterning of sensors on curved surfaces, such as bearings and shafts. The direct-write approach enables cost reductions for patterning of individualized products, such as medical implants.

Key words: strain sensor, thin film, picosecond laser, ablation, 3D-surface

\section{Introduction}

Thin film sensors deposited directly on surfaces of a work-piece have been intensively researched and offer good stability against harsh environmental conditions such as temperatures up to $700^{\circ} \mathrm{C}[1,2,3]$. Patterning of the sensor structures is mostly performed by photolithography using contact or shadow masks. Although, patterning has been demonstrated on 3D-surfaces using flexible masks, thin film sensors on curved surfaces are only used for experimental purposes. Consequently, in serial manufacturing, thin film strain gauges are restricted to flat surfaces, e.g. as pressure sensors, to control the injection pressure in combustion engines.

Laser patterning allows for patterning of thin film strain gauges on curved surfaces and has been demonstrated almost ten years ago [4]. Although, laser patterning has been proved to be a flexible patterning tool, the throughput was too low, to allow for cost effective patterning due to the commercial available beam sources at that time.

Recent developments in the field of laser technology, especially for femtosecond and picosecond lasers lead to an increased availability of robust and stable beam sources, which are required for industrial application.

\section{Fundamentals}

The pulse duration of laser radiation governs the heat penetration depth $L_{\text {therm }}$.

The heat penetration depth of pulses with durations in the range of microseconds and nanosecond can be calculated by

$L_{\text {therm }}=2 \sqrt{D \tau_{\text {pulse }}}=2 \sqrt{\frac{\kappa \tau_{\text {pulse }}}{\rho c}}$

with thermal diffusivity $D$, thermal conductivity $\kappa$, density $\rho$, heat capacity $c$ and pulse duration $\tau_{\text {pulse }}$ [5]. The heat penetration depth in $\mathrm{Ni}$ for a pulse duration of $20 \mathrm{~ns}$ is about $680 \mathrm{~nm}$ and therefore larger than the typical film thickness of strain sensors, which is in most cases smaller than $200 \mathrm{~nm}$. Consequently, a large amount of the absorbed energy is transported into the isolation film and the substrate. The transport of absorbed energy results in heating of the whole thin film system and leads to a high risk of damaging the thin film system, for example through thermally induced stress. The risk of damage through stress increases due to accumulated heat, if the patterning velocity is increased, e.g. by raising the repetition rate of the laser pulses.

The heat penetration depth of pulses of several picoseconds or shorter cannot be calculated by simple thermodynamics. Due to the short pulse duration the energy is absorbed by conducting 
electrons much faster than it can be transferred to the lattice. In order to calculate the heat penetration depth the two-temperature-model, that takes into account the temperatures of the electrons and the lattice, has to be considered. Deriving form the two-temperature model, the heat penetration depth can be calculated by

$L_{\text {therm }}=\sqrt[8]{\frac{128}{\pi}} \sqrt[4]{\frac{\kappa_{\mathrm{e}}^{2} C_{\mathrm{l}}}{T_{\mathrm{m}} A_{\mathrm{e}} g^{2}}}$

with the thermal conductivity of the electrons $\kappa_{\mathrm{e}}$, the heat capacity of the lattice $C_{1}$, the melting Temperature $T_{\mathrm{m}}$, the heat capacity of the electrons $A_{\mathrm{e}}$ and the coupling constant $g$ that describes the heat transfer from the electrons to the lattice [6]. The heat penetration depth in $\mathrm{Ni}$ is about $30 \mathrm{~nm}$ and much smaller than the typical film thickness. Therefore, the thermal impact into the thin film system is significantly reduced and allows for a stable patterning process.

Consequently, pulse durations of a few picoseconds or shorter are to be preferred when patterning thin film sensors. Fundamentals of laser patterning of thin film systems, which are suited for strain sensing, with these pulse durations have been studied $[7,8]$. The developed process allows for fast patterning of thin film strain gauges on 3Dsurfaces [9].

\section{Experimental setup}

The main components of the machining setup used for the patterning of strain sensors are a beam source delivering the pulsed laser radiation ( $\tau_{\text {pulse }} \leq 15 \mathrm{ps}$ ), an $\mathrm{x} / \mathrm{y}$-Scanner for beam deflection and telecentric optics for beam focusing (Fig. 1). The beam radius $w_{0}$ was $12.5 \mu \mathrm{m}$. Patterning was performed without a shift of focus or work piece.

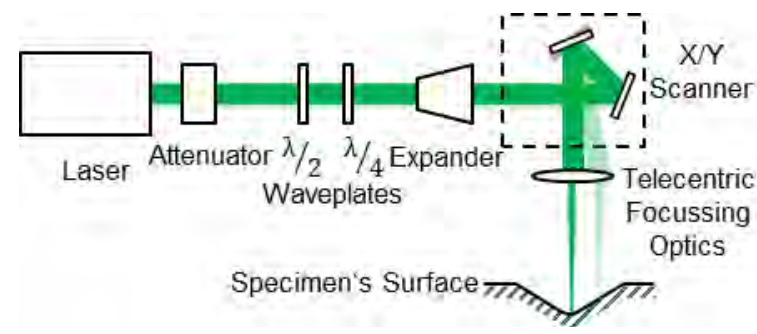

Fig. 1. Machining setup used for the patterning of thin film sensors

\section{Sensors on 3D-surfaces}

Laser patterning on 3D-surfaces was performed on milled v-notched tensile specimen (Fig. 2. a). An $\mathrm{Al}_{2} \mathrm{O}_{3}$-layer of $3 \mu \mathrm{m}$ thickness has been deposited on the milled surface. The insulation film has been deposited on the surface without any polishing steps (Fig. 2. b). The sensor layer is $\mathrm{NiCr}(80 / 20)$ with a thickness of $200 \mathrm{~nm}$. Sensors of two different layouts (Fig. 2. c: A longitudinal and B transversal to the specimen) are used to build a Wheatstone bridge in order to improve the signal/noise-ratio and to compensate thermal disturbance. The sensor layouts consist of meanders with conductor widths of $30 \mu \mathrm{m}$.

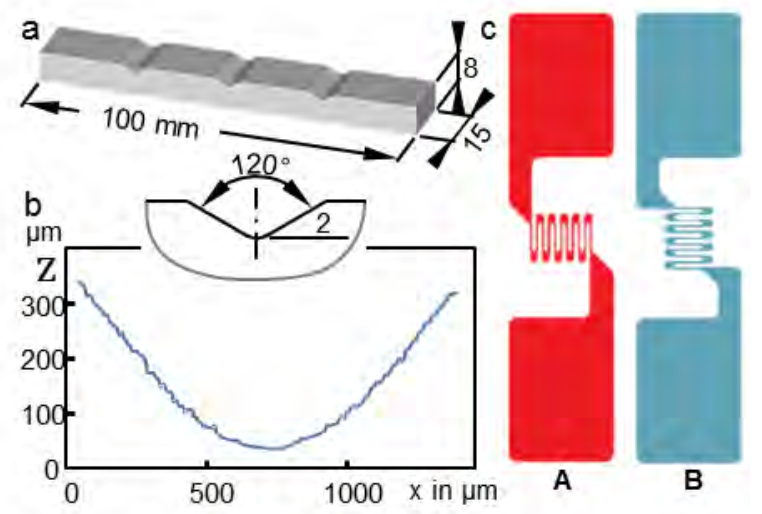

Fig.2. a) V-notched aluminium tensile specimen b) profile of milled $v$-notch c) Layout of strain resistors

Strain gauges are patterned at the bottom of the v-notch (Fig. 3. a). Electrical connection is ensured by conductive adhesive. The areas with removed sensing layer appear black and are approximately $100 \mu \mathrm{m}$ wide. The sensing layer is completely removed in these areas without damaging the insulating film (Fig. 3. b).
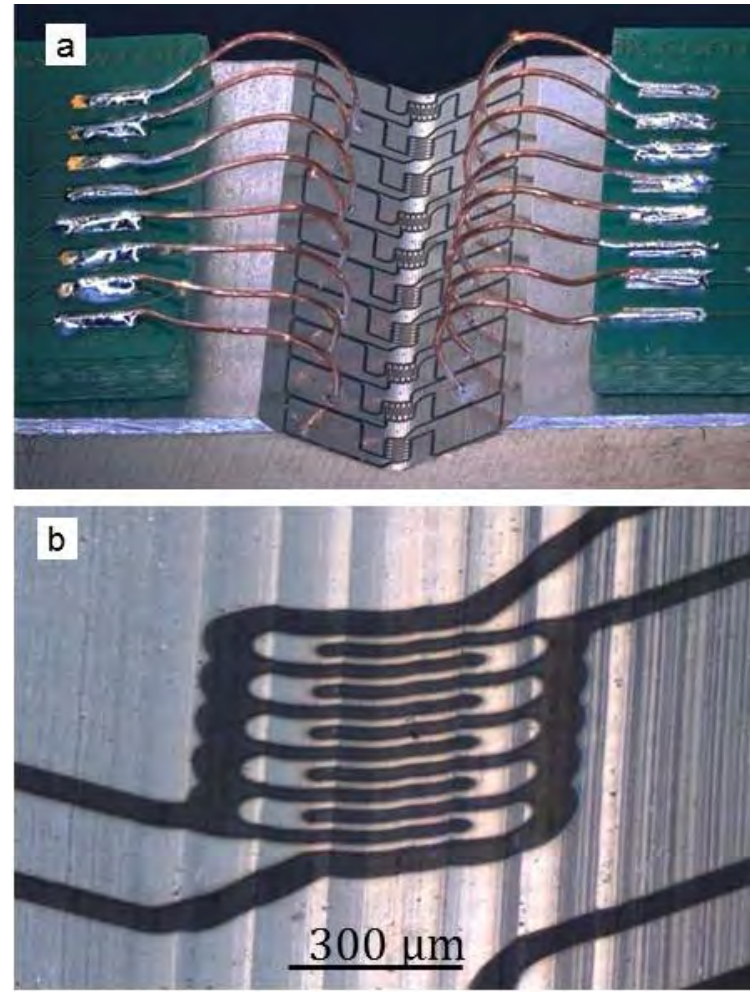

Fig. 3. a) Connected strain sensors for tensile test b) detail of sensor pattern 
The time required to pattern each sensor is less than $15 \mathrm{~s}$. It can be reduced by using higher repetition rates of the beam source. Further details of the process parameters are published in [9].

Four sensor elements (2x longitudinal, 2x tranversal) are interconnected to a Wheatstone bridge (Fig. 4). The offset of the diagonal voltage is compensated by use of an external potentiometer and is amplified by a factor of 500. The Voltage signal of a tensile test, applying a force of $\pm 2.5 \mathrm{kN}$ shows a linear behavior.

Therefore, it can be concluded that the developed laser process is suitable to pattern thin film strain gauges on 3D-surfaces and the patterned sensor exhibit a linear behavior if applied to strain.

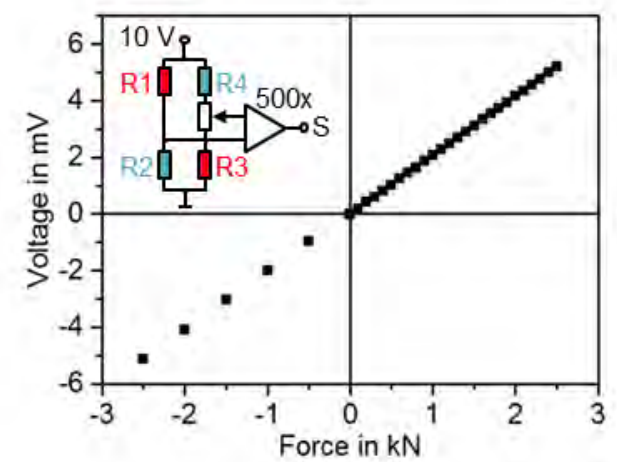

Fig. 4. Results of tensile tests for sensor in v-notch

\section{Influence of conductor width}

In order to study the influence of the patterning process on the sensing material, sensors with conductor widths ranging from $13 \mu \mathrm{m}$ to $73 \mu \mathrm{m}$ are patterned on a flat sample. The resistivity of each sensor element is kept constant by increasing the total sensor length for larger conductor widths (Fig. 5).

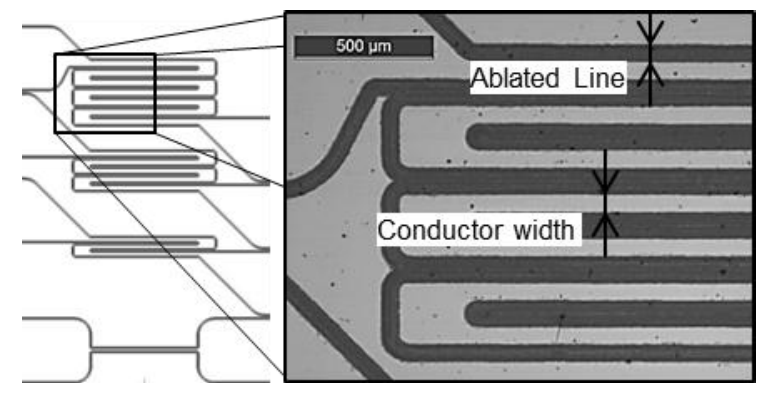

Fig. 5. Sensor layout for the TCR and gauge factor characterization

The TCR is determined by measuring the resistance of sensor elements as a function of the temperature in a range from $10^{\circ} \mathrm{C}$ to $40^{\circ} \mathrm{C}$. The gauge factor is determined measuring the resistance of each sensor element at strains up to $\varepsilon=5.9 \cdot 10^{-4}$.

The TCR of the patterned sensor element is approximately $10^{-6} / \mathrm{K}$ (Fig. 6). A significant influence of the conductor width cannot be identified. The gauge factor of the characterized sensors is approximately 1.8 (Fig. 6) and is in the range expected for the investigated material $(\approx 2)$.

Consequently, it can be concluded that the influence of the patterning with picosecond laser pulses on the properties of the sensing layer can be neglected in the investigated range of conductor widths.

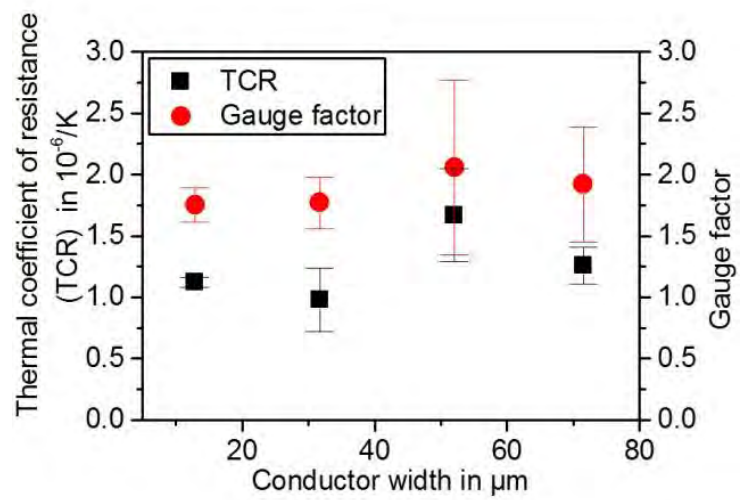

Fig. 6. TCR and gauge factors of sensors with different conductor widths

\section{Conclusion}

Laser ablation with picosecond laser pulses has been proven to be a suitable tool to pattern thin film strain gauges on 3D-surfaces. Experimental studies presented in this paper demonstrate that laser patterned strain sensors in v-notches exhibit a linear strain signal. The laser patterning does not influence the TCR and gauge factor for conducting widths $\geq 13 \mu \mathrm{m}$. Consequently, laser patterning allows for the patterning of miniaturized strain sensors on 3Dsurfaces. The low processing times are ensured by use of galvanometer scanners and will be further reduced by increasing the repetition rate and improving the scan-strategy. The possibility to pattern thin film strain sensors on 3Dsurfaces is a prerequisite to explore new applications, such as load monitoring on bearings and shafts. The direct-write approach results in a significantly simplified process chain in comparison with photolithographic patterning and indicates the possibility to pattern strain sensors for individualized products, such as medical implants.

\section{Acknowledgement}

The authors thank Dr. K.M. Litwinski from IFW Hannover for preparing tension samples, $\mathrm{H}$. 
Gerdes from Fraunhofer IST, Braunschweig, for sputter coating of the samples and T.Mueck for support on TCR and gauge factor measurements. This work was supported by German Research Foundation (DFG) within the Collaborative Research Centre CRC 653, "Gentelligent Components in their Lifecycle"

\section{References}

[1] KAYSER, Godefroy J. C. P.; LeCA, L.: Hightemperature thin-film strain gauges. In: Sensors and Actuators A: Physical 37-38 (1993), S. 328332. doi:10.1016/0924-4247(93)80055-L

[2] LEI, Jih-Fen; WILL, Herbert A.: Thin-film thermocouples and strain-gauge technologies for engine applications. In: Sensors and Actuators $A$ 65 (1998), S. 187-193. doi 10.1016/S09244247(97)01683-X

[3] Mihara, Yuji ; Sekine, Kazuyuki ; Someya, Tsuneo: Study on the development of a thin-film sensor for measuring oil-film pressure in engine bearing and piston pin-boss. In: D. Dowson, G. D. M. Priest P. M. Priest (Hrsg.) ; LUBRECHT, A.A (Hrsg.): Tribological Research and Design for Engineering Systems Proceedings of the 29th Leeds-Lyon Symposium on Tribology Bd. 41. Elsevier, 2003. doi: 10.1016/S01678922(03)80152-9.

[4] BIEHL, S. ; LÜTHJE, H.: Laser patterning of thin films deposited on complex surfaces for the fabrication of micro-sensors. In: GEIGER, M. (Hrsg.); OTTO, A. (Hrsg.): Proceedings of the Lane, 2004

[5] BÄUERLE, Dieter: Nanosecond-Laser Ablation. Version:2011. http://dx.doi.org/. In: Laser Processing and Chemistry. Springer Berlin Heidelberg, 2011. doi: 10.1007/978-3-642-176135_12

[6] Corkum, P. B. ; Brunel, F. ; Sherman, N. K.: Thermal Response of Metals to UltrashortPulse Laser Excitation. In: Phys. Rev. Lett. 61 (1988), December, Nr. 25, 2886-2889. - doi: 10.1103/PhysRevLett.61.2886

[7] Suttmann, Oliver ; Duesing, Jan F. ; Klug, Ulrich ; KLING, Rainer: Patterning of $\mathrm{NiCr}(80 / 20)$ on Al2O3 Using Picosecond Laser Pulses. In: JLMN-Journal of Laser Micro/Nanoengineering 6 (2011), Nr. 1, S. 31-36. doi: 10.2961/jlmn.2011. 01.0008

[8] Duesing, Jan F. ; SUTTMANN, Oliver ; KOCH, Juergen ; StUTE, Uwe ; OVERMEYER, Ludger: Ultrafast Laser Patterning of Thin Films on 3-D Shaped Surfaces for Strain Sensor Applications. In: JLMN-Journal of Laser Micro/Nanoengineering 7 (2012), Nr. 3, S. 311315. doi: 10.2961/jlmn.2012.03.0014

[9] OVermeyer, L. ; Duesing, J.F. ; SuttmanN, O. ; STUTE, U.: Laser patterning of thin film sensors on 3-D surfaces. In: CIRP Annals Manufacturing Technology 61, pp. (2012), Nr. 1, doi: 10.1016/j.cirp.2012.03.087. 